\title{
EFICIÊNCIA DA SELEÇÃO COMBINADA NO MELHORAMENTO GENÉTICO DO TAXI-BRANCO (Sclerolobium paniculatum Vogel)
}

\author{
João Tomé de FARIAS NETO', Alberto William Viana de CASTRO², Silas \\ MOCHIUTTI ${ }^{3}$
}

\begin{abstract}
RESUMO - São apresentados resultados da eficiência da seleção combinada em progênies de meios-irmãos de taxi-branco, com quatro anos de idade, relativamente ao esquema de seleção entre e dentro de familias de meios-irmãos, As 21 progênies foram estudadas em um delineamento de blocos casualisados no Campo Experimental do Cerrado pertencente ao Centro de Pesquisa Agroflorestal do Amapá - Embrapa Amapá, no Estado do Amapá, Brasil. As análises de variâncias apontaram diferenças significativas ao nivel de $1 \%$ de probabilidade para os três caracteres avaliados, indicando serem grandes as chances de obtenção de sucesso com o processo seletivo. Ficou evidenciada a superioridade da seleção combinada sobre a seleção entre e dentro, visto que apresentou ganhos relativos superiores de $30 \%, 42 \%$ e $51 \%$ para DAP, biomassa e altura, respectivamente, indicando ser uma estratégia promissora no melhoramento genético de espécies florestais.
\end{abstract}

Palavras-chave: ganho genético, valor genético, método de seleção, melhoramento florestal.

Efficiency of Combined Selection in Genetic Improvement of Taxi-Branco (Sclerolobium paniculatum Vogel).

ABSTRACT - The efficiency of combined family/within family selection was evaluated in half-sib progenies of taxi-branco (Sclerolobium paniculatum Vogel) at the Cerrado Experiment Station, Embrapa Amapá, Amapá, Brazil, in a four-year-old 21 half-sib progeny trial in a randomized block design. Highly significant differences $(p<1 \%)$ were found for three traits likely to show high genetic gain due to selection: diameter $(\mathrm{DBH})$, biomass and height. Combined selection provided estimates of $30 \%, 42 \%$ and $51 \%$ gains, respectively, confirming that combined selection is a promising strategy in forest tree improvement. Effective population size must be estimated to assure genetic gain and preserve genetic variability.

Key words: genetic gain, genetic value, selection methods, forest tree improvement.

\section{INTRODUÇÃO}

Dentre as espécies arbóreas nativas da região amazônica o taxibranco (Sclerolobium paniculatum Vogel) se destaca pelo rápido crescimento e boa qualidade da madeira para produção de carvão (Castro et al., 1990; Dias et al., 1995), já merecendo inclusive trabalhos na área de melhoramento.
No Brasil o emprego de seleção entre e dentro de famílias de meios-irmãos é o procedimento mais utilizado em programas de melhoramento genético de espécies florestais. Esse esquema se caracteriza por envolver basicamente uma seleção entre as progênies, estando elas representadas na unidade de recombinação pelas suas melhores plantas. $\mathrm{Na}$ prática florestal, esta última etapa consiste em

1 Eng. Agr., Dr., Embrapa/Centro de Pesquisa Agroflorestal do Amapá. (CPAF-AP). Caixa Postal 10, CEP 68902-280, Macapá, AP.

2 Eng. Ftal., M.Sc. Embrapa/Centro de Pesquisa Agroflorestal da Amazônia Oriental (CPATU), Caixa Postal 48, CEP 66017-970, Belém, PA.

3 Eng.Agr., M.Sc,, Embrapa/Centro de Pesquisa Agroflorestal do Amapá (CPAF-AP). Caixa Postal 10, CEP 68902-280, Macapá, AP. 
selecionar a melhor árvore de cada parcela em que a progênie selecionada esteja presente. Tal fato se constitui em limitação desse esquema, visto que árvores excepcionais de progênies não selecionadas (famílias intermediárias e inferiores) são excluídas e também que, dentro das familias selecionadas, o melhor individuo de uma determinada familia muitas vezes é inferior ao segundo, terceiro ou quarto melhores individuos de outras familias (Resende \& Higa, 1994) .

Uma estratégia recentemente sugerida para o melhoramento de espécies florestais envolve o emprego da seleção combinada (Resende \& Higa, 1994; Resende \& Bertolucci, 1993). Nesse tipo de seleção, originalmente proposto por Lush (1947), é determinado o valor genético de todos os indivíduos da população sob seleção, por meio de um indice que reúne informações do próprio individuo e de sua familia (mérito familiar). No Brasil, os poucos resultados disponiveis na literatura têm demonstrado que a utilização da seleção combinada tem sido eficiente no melhoramento de populações florestais. Sobre a eficiência da seleção combinada relativamente a seleção entre e dentro de familias de meiosirmãos, Bueno Filho (1992) reportou ganhos genéticos superiores de $25 \%$ para altura, $27 \%$ para diâmetro à altura do peito (DAP) e $24 \%$ para volume aos três anos de idade em E. grandis. Aos sete anos o mesmo autor estimou ganhos superiores de $27 \%$ para altura e $31 \%$ para DAP e volume. Pires et al. (1996) também reportam ganhos superiores de $20,14 \%$ para DAP e $14,73 \%$ para altura com o emprego da seleção combinada. Resende \& Higa (1994) alcançaram ganhos de $30 \%$ e $35 \%$ para altura e DAP, respectivamente em $E$. grandis.

Assim sendo, o presente trabalho teve como objetivo comparar os ganhos genéticos resultantes da aplicação da seleção combinada e da seleção entre e dentro de famílias de meios-irmãos em taxi-branco para os caracteres altura, DAP e biomassa.

\section{MATERIAL E MÉTODOS}

Foram utilizadas progênies de meios-irmãos de taxi-branco obtidas de árvores selecionadas na Floresta Nacional do Tapajós (FLONA), localizada no município de Belterra, Estado do Pará. Na seleção fenotipica das árvores utilizaramse como critérios, características de crescimento em altura, DAP e volume. Foram coletadas sementes de 21 árvores, as quais foram utilizadas para instalação do experimento.

$\mathrm{O}$ experimento foi instalado em 1989, no Campo Experimental do Cerrado, pertencente ao Centro de Pesquisa Agroflorestal do Amapá-CPAF-Amapá, situado a $0^{\circ} 22^{\prime} \mathrm{Ne} 51^{\circ} \mathrm{W}$ e 50 metros de altitude, aproximadamente a $45 \mathrm{Km}$ de Macapá. Aárea apresenta topografia plana, cobertura vegetal de cerrado, clima tipo Ami segundo a classificação de Köppen, temperatura média anual de $27^{\circ} \mathrm{C}$, umidade média relativa do ar de $85 \%$ e precipitação média anual de $2.300 \mathrm{~mm}$ (Boletim, 1990). O solo é do tipo Latossolo Amarelo, textura média e de baixa fertilidade (Tab. 1).

Foram empregadas dez repetições em delineamento de blocos casualisados, com as parcelas experimentais lineares e representadas por cinco plantas, ao espaçamento de $3,0 \times 3,0 \mathrm{~m}$. O experimento 
Tabela 1. Caracteristicas físicas e quimicas do solo.

\begin{tabular}{|c|c|c|c|c|c|c|c|c|c|c|c|}
\hline \multirow{2}{*}{$\begin{array}{l}\text { Prof. } \\
(\mathrm{cm})\end{array}$} & \multirow{2}{*}{$\begin{array}{l}\text { Areia } \\
(\%)\end{array}$} & \multirow{2}{*}{$\begin{array}{l}\text { Silte } \\
(\%)\end{array}$} & \multirow{2}{*}{$\begin{array}{l}\text { Argila } \\
(\%)\end{array}$} & \multirow[t]{2}{*}{$\mathrm{pH}$} & \multirow{2}{*}{$\begin{array}{l}\text { M.O } \\
(\%)\end{array}$} & \multirow{2}{*}{$\begin{array}{l}\mathrm{P} \\
\text { (ppm) }\end{array}$} & \multicolumn{4}{|c|}{$\mathrm{CTC}(\mathrm{meq} / 100 \mathrm{ml})$} & \multirow{2}{*}{$\begin{array}{l}\text { Sat.AI } \\
(\%)\end{array}$} \\
\hline & & & & & & & Al & $\mathrm{Ca}$ & $\mathrm{Mg}$ & K & \\
\hline $0-20$ & 68 & 9 & 23 & 4,5 & 0,98 & 0,9 & 0,86 & 0,08 & 0,04 & 0,03 & 85,1 \\
\hline
\end{tabular}

foi avaliado no campo aos 48 meses após o plantio, coletando-se dados de crescimento em altura e DAP. O caráter biomassa (BMS) ${ }^{1}$ foi obtido por meio da expressão $\mathrm{BMS}=-3.64+0,593 \times \mathrm{CSA}$ onde: $\mathrm{CSA}=$ somatório dos diâmetros em centimetros ao quadrado dos fustes numa mesma árvore (árvores com mais de um fuste) à altura de $1,30 \mathrm{~m}$. A coleta dos dados para os caracteres altura, DAP e biomassa foi feita ao nível de plantas individuais.

A análise de variância foi realizada segundo o delineamento de blocos casualisados, empregando o programa computacional PAGIS (Programa de análise genética e índice de seleção) desenvolvido por Oliveira et al. (1994). A partir destas análises foram estimados os ganhos genéticos esperados com o emprego da seleção entre e dentro de famílias de meios-irmãos e combinada.
Detalhes sobre aplicação da seleção combinada foram apresentados por Bueno Filho (1992), Resende \& Higa (1994) e Pires et al. (1996). Foram empregadas intensidades de seleção entre de $30 \%$ (i $=1,16)$ e dentro de $20 \%(\mathrm{i}=1,40)$. A efíciência da seleção combinada sobre a seleção entre e dentro foi estimada segundo procedimento apresentado por Bueno Filho (1992).

\section{RESULTADOS E DISCUSSÃO}

$\mathrm{Na}$ tabela 2 são apresentados os valores e significâncias dos quadrados médios (Teste F) obtidos nas análises de variấncias, Foram detectadas diferenças significativas para os três caracteres ao nivel de $1 \%$ de probabilidade, indicando perspectivas promissoras de obtenção de ganhos com o processo seletivo. Os coeficientes de variação experimental

Tabela 2. Quadrados médios obtidos nas análises de variâncias para três caracteres em progênies de taxi-branco aos quatro anos. Macapá, AP.

\begin{tabular}{lllll}
\hline & FV & GL & \multicolumn{3}{c}{ Quadrados Médios } \\
\cline { 3 - 5 } & & Altura & DAP & Biomassa \\
\hline Blocos & 09 & 0,559 & 1,971 & 124,403 \\
Tratamentos & 20 & $0,451^{\star \star}$ & $0,934^{\star \star}$ & $178,401^{\text {** }}$ \\
Entre & 180 & 0,134 & 0,292 & 35,366 \\
Dentro & 840 & 0,526 & 1,148 & 135,751 \\
Média Geral & & 3,92 & 4,19 & 17,85 \\
CV (\%) & & 9,34 & 12,86 & 33,31 \\
\hline
\end{tabular}

** : significativo a $1 \%$ de probabilidade.

1 Informação pessoal prestada pelo Dr. Milton Kanashiro (Embrapa Amazônia Oriental) 
para altura, DAP e biomassa indicam um nível de precisão considerado de média magnitude por Garcia (1989) em estudos com espécies florestais.

$\mathrm{Na}$ tabela 3, encontram-se as estimativas dos progressos esperados com o emprego da seleção entre e dentro de familias de meios-irmãos e combinada. Observa-se que para todos os caracteres foram estimados ganhos superiores com o emprego da seleção combinada quando comparados com a seleção entre e dentro. Em termos relativos, o maior progresso estimado ocorreu para o caráter altura $(51 \%)$, seguido pela biomassa $(42 \%)$ e DAP $(30 \%)$. Esses resultados estão coerentes com os encontrados na literatura com outras espécies florestais. Dessa forma, o uso da seleção combinada no melhoramento genético do taxi-branco seria uma alternativa interessante a ser recomendada.

Tabela 3. Estimativas dos progressos genéticos esperados $(\%)$ para seleção entre e dentro e combinada em progênies de taxi-branco aos quatro anos. Macapá, AP.

\begin{tabular}{lccc}
\hline PROGRESSOS & ALTURA & DAP & BIOMASSA \\
\hline Gs entre & 4,42 & 3,82 & 8,43 \\
Gs dentro & 4,61 & 6,01 & 28,80 \\
Gs total & 9,03 & 9,83 & 37,23 \\
G $_{s}$ combinada & 13,62 & 12,78 & 53,23 \\
Eficiência relativa & 1,51 & 1,30 & 1,42 \\
\hline
\end{tabular}

As progènies selecionadas pela seleção combinada encontram-se na tabela 4. Os valores médios estimados das árvores selecionadas foram de $5,33 \mathrm{~m}$ para altura, $6,75 \mathrm{~cm}$ para DAP e $53,33 \mathrm{~kg} /$ planta para biomassa. Nota-se ainda que nas progênies de menor média foram apenas selecionadas árvores com desempenho excelente, resultado do elevado mérito familiar. Para o caráter altura, nota-se que a progênie de número 3 apresentou uma média intermediária $(3,92 \mathrm{~m})$, porém foi identificada uma árvore de ótimo desempenho $(6,42 \mathrm{~m})$. Com relação ao DAP, a progênie de número 11 apresentou média intermediária de $4,08 \mathrm{~cm}$, porém foi identificada uma árvore também de ótimo desempenho com $9,39 \mathrm{~cm}$ de DAP.

Considerando-se o caráter biomassa, a progênie de número 02 teve média também, intermediária $(19,20 \mathrm{~kg} /$ planta $)$, sendo identificada uma árvore de maior produtividade $(73,32 \mathrm{~kg} /$ planta) em todo o experimento. Entretanto, vale ressaltar que a seleção combinada tende a selecionar um grande número de indivíduos de determinadas familias, em função do maior peso dado à informação de familia, como é o caso da familia de número 01 (15 indivíduos selecionados) para altura, familia número 21 (19 individuos selecionados) para DAP e família número 01 (14 indivíduos selecionados) para biomassa. Como conseqüência é recomendável estimar o tamanho efetivo populacional associado a cada esquema de seleção, de modo a maximizar o progresso genético e manter a variabilidade para seleção a médio e longo prazo.

\section{CONCLUSÕES}

Para os três caracteres estudados, a seleção combinada demonstrou ser um eficiente método a ser empregado no melhoramento genético do taxi-branco, haja vista que aferiu ganhos genéticos superiores relativamente ao esquema de seleção entre e dentro de famílias de meios irmãos ( $30 \%$ para DAP, $42 \%$ para biomassa e $51 \%$ para altura). 
Tabela 4. Estimativas dos valores médios para famílias, familias selecionadas e número de árvores selecionadas de taxi-branco aos quatro anos de idade, empregando-se a seleção combinada. Macapá, AP.

\begin{tabular}{|c|c|c|c|c|c|c|c|c|c|}
\hline \multirow[b]{2}{*}{ FAM. } & \multirow[b]{2}{*}{ Familia. } & \multicolumn{2}{|l|}{ ALTURA (m) } & \multicolumn{3}{|c|}{ DAP (cm) } & \multicolumn{3}{|c|}{ BIOMASSA (kg/planta) } \\
\hline & & Selecionada & № Árvores & Familia & Selecionada & № Árvore & Familia & Selecionada & $\mathrm{N}^{2}$ Árvores \\
\hline 01 & 4,49 & 4.91 & 15 & 4,71 & 5,62 & 15 & 26,28 & 41,68 & 14 \\
\hline 02 & 3,96 & 6,00 & 01 & 4,17 & 7,71 & 02 & 19,20 & 73.32 & 01 \\
\hline 03 & 3,92 & 6,42 & 01 & 4,26 & - & - & 17,17 & - & - \\
\hline 04 & 4,12 & 5,47 & 05 & 4,42 & 7,01 & 03 & 21,01 & 42,77 & 04 \\
\hline 05 & 4,11 & 5,28 & 06 & 4,33 & 6,33 & 02 & 23,79 & 49,78 & 08 \\
\hline 06 & 4,10 & 5,60 & 02 & 4,42 & 6,64 & 04 & 24,46 & 43,47 & 12 \\
\hline 07 & 4,13 & 5,13 & 21 & 4,36 & 6,69 & 02 & 19,12 & 49,94 & 01 \\
\hline 08 & 3,81 & - & - & 4,15 & 6,82 & 01 & 14,27 & - & . \\
\hline 09 & 3,84 & - & - & 4,54 & 6,60 & 06 & 14,76 & - & - \\
\hline 10 & 4,12 & 5,55 & 02 & 4,20 & - & - & 20,35 & 61,35 & 01 \\
\hline 11 & 3,88 & 6,60 & 02 & 4,08 & 9,39 & 01 & 19,94 & 49,20 & 02 \\
\hline 12 & 3,80 & - & - & 4,42 & 5,83 & 02 & 11,60 & - & , \\
\hline 13 & 3,97 & 5,85 & 01 & 4,31 & 6,33 & 03 & 18,03 & 41,00 & 02 \\
\hline 14 & 3,49 & - & - & 3,68 & - & - & 12,11 & . & - \\
\hline 15 & 3,91 & - & $=$ & 4,15 & - & - & 10,02 & 54,86 & 03 \\
\hline 16 & 3,85 & 6,60 & 01 & 3,86 & - & - & 15,97 & 68,37 & 01 \\
\hline 17 & 3,61 & - & - & 3,77 & - & - & 15,15 & 64.81 & 02 \\
\hline 18 & 3,75 & - & - & 3,87 & - & - & 14,17 & 3 & - \\
\hline 19 & 3,77 & - & - & 4,04 & - & - & 13,63 & - & - \\
\hline 20 & 3,67 & - & - & 3,66 & - & . & 12,73 & 63,58 & 01 \\
\hline 21 & 3,94 & 5,81 & 01 & 4,73 & 6,05 & 19 & 22,10 & 42,52 & 08 \\
\hline Médias & 3,92 & 5.33 & & 4,19 & 6,75 & & 17,85 & 53,33 & \\
\hline
\end{tabular}

\section{Bibliografia Citada}

Boletim Agrometeorológico.1990. Macapá: EMBRAPA-UEPAE de Macapá, 55p.

Bueno Filho, J.S.S. 1992. Seleçào combinada versus seleção sequencial no melhoramento de populações florestais. Piracicaba, SP: ESALQ/ USP. Dissertação apresentada a Escola Superior de Agronomia Luiz de Queiroz da Universidade de São Paulo, SP, 96 p.

Castro, A. W.V.; Yared, J.G.; Alves, R.N.B.; Silva, L.S.; Meirelles, S.M.L.B. 1990. Comportamento silvicultural de Sclerolobium paniculatum (taxi-branco) no cerrado amapaense. Macapá: EMBRAPAUEPAE de Macapá, 4p. (EMBRAPA-UEPAE DE Macapá, Comunicado técnico, 7)

Dias, L.E.; Brienza Junior, S.; Pereira, C. A. 1995. Taxi-branco (Sclerolobium paniculatum Vogel): uma leguminosa arbórea nativa da Amazônia com potencial para recuperação de áreas degradadas. In: Kanashiro, M.;
Parrota, J.A (ed) Manejo e reabilitação de áreas degradadas e florestas secundárias na Amazônia. Paris, França: UNESCO, p. 148-153.

Garcia, C.H. 1989. Tabelas para classificação do coeficiente de variação. Piracicaba, SP: IPEF, 11p. (IPEF, circular técnica, 171).

Lush, J. L. 1947. Family merit and individual merit as basis for selection. American Naturalist, v. 80 , p. $318-342$.

Oliveira, E. B. de; Resende, M.D.V.; Higa, A. R.; Oida, G.R.P. 1994. PAGIS - um software para análise genética e indice de seleção em experimentos florestais. Colombo, PR: EMBRAPA-CNPF, 19p. (EMBRAPA-CNPF, Documentos, 24).

Pires, E. I.; Cruz, C. D.; Borges, R.C.G.; Regazzi, A J. 1996. Indice de seleção combinada aplicado ao melhoramento genético de Eucalyptus spp. Revista Arvore, Viçosa, v. 20, n. 2, p. 191-197.

Resende, M.D.V. de; Higa, A R. 1994. Estimação 
de valores genéticos no melhoramento de Eucalyptus: seleção em um caráter com base em informações do individuo e de seus parentes, Colombo, PR: EMBRAPACNPF, p.11-36 (EMBRAPA-CNPF, Boletim de Pesquisa Florestal, 228/29).

Rezende, G.D.S.P.; Bertolucci, F.L.G. 1993. Uso da seleção combinada na determinação da eficiência da seleçâo precoce em progênies de meio-imãos de Eucalyptus urophylla, Anais Congresso Florestal Pan-Americano, I e Congresso Florestal Brasileiro, 7, p.158-160. 